\title{
ORIGINAL
}

\section{Classification of physical activity in patients with heart failure categorized as New York Heart Association class I or II}

\author{
Sayuri Doi ${ }^{1}$, Ayako Tamura², Takako Minagawa², Akemi Osaka³ ${ }^{3}$ and Masataka Sata ${ }^{4}$ \\ ${ }^{1}$ Tokushima University Graduate School of Health Sciences, Doctor Course Student, Tokushima, Japan, ${ }^{2}$ Department of Nursing, Tokushima \\ University Graduate School of Biomedical Sciences, Tokushima, Japan, ${ }^{3}$ Tokushima University Hospital, Tokushima, Japan, ${ }^{4}$ Department of \\ Cardiovascular Medicine, Tokushima University Graduate School of Medicine, Tokushima, Japan
}

\begin{abstract}
This study aimed that we were classification of physical activity in patients with heart failure categorized as New York Heart Association (NYHA) class I or II. We were a survey using a researcher- administered questionnaire, SF-8, the Specific Activity Scale (SAS), and the Scale to Measure Self-Care Behavior of Patients with Heart Disease. We included 70 patients who were treated in the Department of Cardiovascular Medicine at Hospital A. Regarding patient characteristics and clinical information after the cluster analysis, there were significant differences in the NYHA class $(p=0.001)$, BNP level $(p=0.012)$, self-management of medication adherence $(p=0.000)$, and exercise habits $(p=0.005)$. We summarized characteristics of each group as follows : Group A showed high tolerance to physical activity and near-perfect self-management; Group B showed moderate tolerance to physical activity but was not willing to commit to daily exercise and self-management; and Group $C$ showed low tolerance to physical activity and often requested others to handle medication management. We needed that tolerance to physical activity and proposals for tailored instruction according to patient conditions, and needed that instructions tailored to the characteristics of heart failure patients in groups A-C. J. Med. Invest. $67: 124-133$, February, 2020
\end{abstract}

Keywords : classification of physical activity, patients with heart failure, NYHA

\section{INTRODUCTION}

Many patients with heart failure are repeatedly readmitted due to the aggravation of their disease (1), and the number of patients with heart failure has been increasing every year (2). In addition, patient's vital prognosis remains extremely poor (3). However, healthy life expectancy in these patients can be extended through continuous physical activity along with symptom control in daily life.

A previous study showed that medication compliance and strict salt restriction are the key strategies for symptom control (4). Another previous study indicated that patients should pay attention to changes in their physical condition to prevent aggravation of symptoms (5). Regarding physical activity in daily life, some studies evaluated levels of independent walking at discharge (6) and ability to perform activities of daily living 2 months after discharge (7) and performed qualitative analyses of changes in patients' physical condition in daily life (8). However, there has been no study focusing on factors associated with physical activity. Overseas studies have identified physical activity as a factor related to education and age (9), but not for patients with heart failure.

To prevent aggravation of symptoms in patients with heart failure, instructions on physical activity should be tailored to each patient. Most previous studies have focused on medication compliance and strict salt restriction, both of which are associated with improvement of symptoms; however, no study has evaluated adherence to physical activity (active commitment).

Received for publication December 6, 2019 ; accepted January 8, 2020.

Address correspondence and reprint requests to Sayuri Doi, Tokushima University Graduate School of Health Sciences, Doctor Course, 3-18-15, Kuramoto- cho, Tokushima 770-8503, Japan.
Methods for optimizing physical activity should be offered according to the condition of each patient.

We classified factors associated with physical activity in patients with heart failure due to cardiovascular diseases in order to help medical professionals better instruct patients on the basis of these factors.

\section{METHODS}

1. Study design : a cross sectional study using a researcher-administered questionnaire (i.e., interview)

2. Study period: July 31, 2018, to August 31, 2019

3. Patient characteristics :

1) Selection criteria were as follows :

(1) Outpatients and inpatients treated in the Department of Cardiovascular Medicine at Hospital A

(2) Patients (males and females) with chronic heart failure diagnosed by a cardiologist

(3) Patients categorized as having New York Heart Association (NYHA) class I or II heart failure

(4) Patients aged $\geq 40$ years and $\leq 90$ years

2) Recruitment and exclusion criteria :

We recruited in- and outpatients who were referred by cardiologists and a head nurse.

Exclusion criteria were as follows : heart failure patients with a genetic or congenital causal hearth disease; patients who were hospitalized and received treatment for other severe diseases ; and patients with a Hasegawa Dementia Scale score of $\leq 20$.

4. Questionnaire :

The questionnaire comprised five sections as follows : 
1) Questionnaire 1 was prepared by the authors based on the Guidelines for Treatment of Chronic Heart Failure and comprised questions on patient characteristics (10). Items in this questionnaire included age, sex, family structure, participation in social activities, frequency of blood pressure and weight measurements, attitudes on salt restriction, frequency of exercise, and use of Shinfuzen Techou [Heart Failure Booklet] published by the Japanese Heart Failure Society.

2) Questionnaire 2 was prepared by the authors based on the Guidelines for Treatment of Chronic Heart Failure and comprised questions on clinical information (10). This information was collected from the patients' electronic hospital files, which included diagnosis, underlying heart diseases, onset date of the underlying heart disease, number of hospitalizations due to the heart disease, and the presence or absence of complications and their diagnoses. Levels of brain natriuretic peptides (BNPs ; cutoff $=18.4 \mathrm{pg} / \mathrm{mL}$ ), left ventricular ejection fraction (LVEF ; heart failure with a preserved LVEF of $50 \% \geq$ ), and NYHA class, which are used by nurses in patient education (11), were used to assess cardiac function. Other items in the questionnaire comprised blood pressure, pulse, height, and weight, type of pharmacotherapy, and history of smoking and drinking alcohol at the time of the survey.

3) Questionnaire 3 used was based on the Health-Related Quality of Life (QOL) Scale (SF-8) devised by Fukuhara et al. (12). We used this scale because patients with chronic disease may show poor QOL. This questionnaire evaluated the following eight items : physical function (PF), role physical (RP), bodily pain (BP), general health (GH), vitality (VT), social functioning (SF), role emotional (RE), and mental health $(\mathrm{MH})$. Responses from the patients were scored. The total score ranged from 0 to 100, with a higher score representing better Health-Related QOL. The score was calculated using the SF- ${ }^{\mathrm{TM}}$ scoring program (2_1) (13). The reliability coefficient (Cronbach's alpha) was 0.65 .

4) Questionnaire 4 was based on the Specific Activity Scale (SAS) devised by Sasayama et al. (14). This scale assesses tolerance to performing physical activity in daily life. The scale comprised 21 questions such as "do you fall asleep easily at night?" and "does your condition become bad after doing jump rope?" and responders selected one of the following answers : "Yes," "It is strenuous," and "I do not know" "Yes" was 3 points in the section on physical activity, "It is strenuous" was 2 points in the section regarding activities that patients cannot tolerate, and "I do not know" was 1 point).

5) Questionnaire 5 was based on the Scale to Measure SelfCare Behavior of Patients with Heart Disease devised by Yoshie et al. (15). Questions in this questionnaire evaluated the point to which patients could manage activities of daily life independently. It comprised 20 questions querying adjustments to their mental conditions (4 questions), planning of daily exercise (4 questions), adjustments to sleep and rest (4 questions), salt restriction (4 questions), and prevention for infection (4 questions). The responses were scored on a 5 -point Likert scale, with a higher score indicating better self-management : $1:$ not true at all, 2 : not true ; 3 : somewhat true; $4:$ true; 5 : completely true. Three questions used an inverted scale. The reliability coefficient (Cronbach's alpha) was 0.782 .

\section{Data collection and interview duration}

Data were collected after obtaining informed consent from all participants. Outpatients were interviewed in the treatment room and inpatients were interviewed in the patients' room, counseling room, or treatment room to protect their privacy. A researcher performed an interview using the questionnaires and filled in the answers on the questionnaires. The interview time was approximately $25-30$ minutes.

\section{Data analyses}

Data on patient characteristics, clinical information, SF-8, and Scale to Measure Self-Care Behavior of Patients with Heart Disease results were compiled by simple tabulation. Next, we focused on similarities between 21 responses to the SAS and the level of tolerance to physical activity in daily life. We performed a hierarchical cluster analysis using Ward's method to classify these responses. The number of clusters was determined with a dendrogram. Heart failure patients' characteristics, clinical information, SF-8 results, and Scale to Measure Self-Care Behavior of Patients with Heart Disease results were classified into three groups using cluster analysis for inter-group comparison. Qualitative data were analyzed using chi-squared test and Fisher's exact test, and quantitative data were analyzed using nonparametric methods (Kruskal-Wallis test and Dann-Bonferroni's adjustment for multiple comparisons). SPSS version 24 was used for all analyses, and statistical significance was set at $<5 \%$.

\section{Ethical considerations}

This study was approved by the ethics committee of Tokushima University Hospital (approval number : 3238). A summary of this study was provided verbally and in writing to the head of the medical department and head nurses of the outpatient and inpatient sections, who approved the protocol of this study.

The summary included the following: (a) participation in this study is voluntary; (b) non-participation will not result in disadvantages to the patient; (c) responses to the questionnaires will be anonymous ; (d) patients' data will be anonymized before being subjected to statistical analysis ; (e) the completed questionnaires will be shared only with researchers and will be strictly stored and managed ; (f) after analyzing the responses to these questionnaires, they will be shredded ; and (g) the data obtained from this study will be presented at scientific conferences.

After confirming that the patient completely understood their rights and responsibilities, written consent was obtained. For outpatients, consent was obtained during the waiting time after treatment and for inpatients, consent was obtained during times that did not affect their treatment and care to reduce physical and mental burden. All scales were used after obtaining permission from the developers.

\section{RESULTS}

\section{Summary of patient characteristics (Table 1)}

We included 70 patients with heart failure who provided consent. Median age of the 70 patients was 72.0 (63.0-80.0) years. There were 45 males ( $64.2 \%)$, and 60 patients were living with a co-resident (85.7\%). Twenty-six patients had a job (37.1\%) and five patients (7.1\%) were engaged in community activities. Regarding the breakdown of the underlying heart diseases (3) causing heart failure, ischemic heart disease and cardiomyopathy-which are myocardial abnormalities-occurred in 36 patients (51.4\%). Hypertension and valvular disease/structural abnormality-which are hemodynamic abnormalities-occurred in 16 patients (22.9\%). Arrhythmia was observed in 18 $(25.7 \%)$ patients. Median disease duration after the onset of the underlying heart disease was 41.5 (12-109.3) months. Median number of hospitalizations due to heart diseases was 2 (1-3).

Moreover, 36 (51.4\%) patients presented with NYHA class I and $34(48.6 \%)$ with class II. Median BNP level was 95.3 (36.1393.6) $\mathrm{pg} / \mathrm{mL}$, with a slightly high cutoff value. Median LVEF was $61 \%(51.3 \%-65.8 \%)$. There were $65(92.9 \%)$ patients with a history of one of the following diseases : diabetes, hypertension, 
Table 1. Sammary of patient characteristics $(\mathrm{N}=70)$

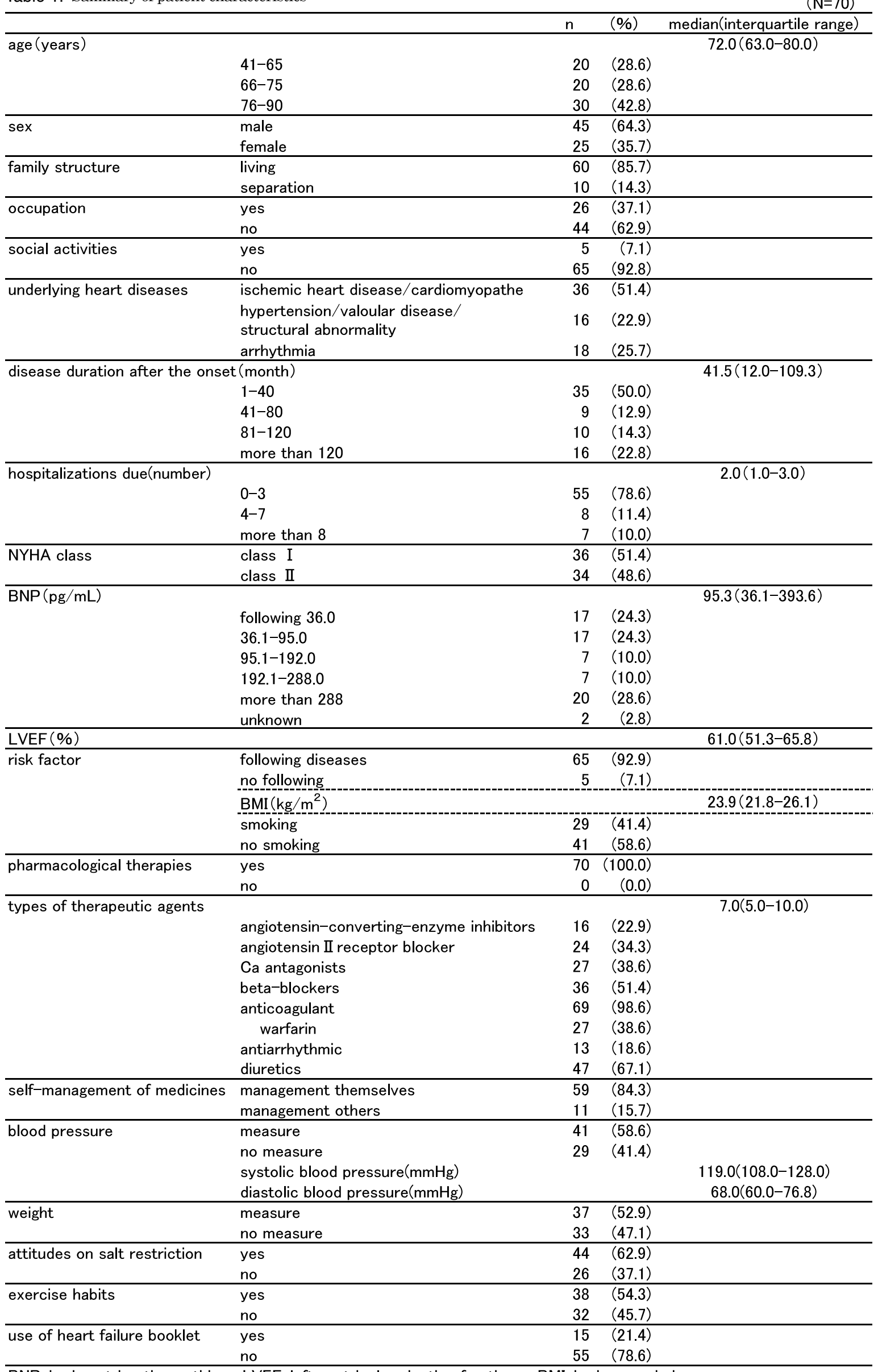

BNP : brain natriuretic peptide

LVEF : left ventricular ejection fraction

BMI : body mass index 
and dyslipidemia, which are risk factors for heart disease. Median body mass index (BMI) was 23.9 (21.8-26.1) $\mathrm{kg} / \mathrm{m} 2$, and 29 (41.4\%) patients were smokers.

All patients (70 patients) received pharmacological therapies. Median number of agents received per day was 7 (5-10). The breakdown of these agents was as follows : 16 (22.9\%) patients received angiotensin-converting-enzyme inhibitors, 27 (38.6\%) received $\mathrm{Ca}$ antagonists, 36 (51.4\%) received beta-blockers, 27 (38.6\%) received warfarin, $13(18.6 \%)$ received antiarrhythmic agents, and $47(67.1 \%)$ received diuretics.

A total of 59 (84.3\%) patients performed medication management themselves. Respectively 41 (58.1\%) and 37 (52.9\%) patients measured blood pressure and weight. Moreover, 44 $(62.9 \%)$ patients were aware of salt restriction, and 38 (54.3\%) performed regular exercise. Median systolic blood pressure was 119 (108-128) $\mathrm{mmHg}$, which was within the range of optimal blood pressure.

\section{Results of the Health-Related QOL Scale (Table 2)}

The Health-Related QOL Scale scores (eight items) for bodily pain $(\mathrm{BP})(50.23 \pm 8.41)$ and mental health $(\mathrm{MH})(50.15 \pm 6.08)$ were higher than Japanese standards, although the differences were negligible. Scores for the other six items in this scale were lower than standards by $2-10$ points, particularly for physical functioning $(\mathrm{PF})(39.94 \pm 9.92)$ and general health $(\mathrm{GH})$ $(42.16 \pm 8.97)$, which were $7-10$ points lower than the standard values.

\section{Results of the Scale to Measure Self-Care Behavior of Patients} with Heart Failure (Table 3)

Score for the Scale to Measure Self-Care Behavior of Patients with Heart Disease in heart failure patients was the highest for the item "listening to opinions from others with a calm mind without getting upset" $(4.20 \pm 0.44)$ and the lowest for the item "taking a nap every day" $(2.16 \pm 0.73)$. Score for items related to adjustments of mental conditions, such as "trying not to be irritated" $(4.10 \pm 0.59)$ and "trying not to think too much" $(4.04 \pm 0.71)$ were generally high. However, scores for items related to physical activity, such as "going for a stroll" ( $2.87 \pm 1.21)$ and "doing exercise to the radio or exercise by oneself every day" $(2.73 \pm 1.20)$, were generally low.

4. Cluster analysis results based on tolerance to physical activity in daily life (Figure 1 and Tables 4-6)

A cluster analysis was performed on SAS scores regarding tolerance to physical activity in daily life using Ward's method. The resulting scores were classified into three groups according to tolerance to physical activity based on a dendrogram (Figure 1).

The cluster analysis indicated that patient characteristics and clinical symptoms (Table 4 ) in groups A-C were significantly different in terms of NYHA class $(p=0.001)$, BNP level $(p=0.012)$ self-management of medication adherence $(p=0.000)$, and exercise habits $(p=0.005)$. There were no significant differences observed in disease duration, number of hospitalizations, LVEF, and number of heart failure drugs.

Cluster analysis of Health-Related QOL Scale scores in groups A-C (Table 5) showed significant differences in terms of PF $(p=0.027), R P(p=0.034), G H(p=0.007)$, and VT $(p=0.041)$. Scores of self-care scale (Table 6) indicated significant differences in "going for a stroll" $(p=0.000)$, "going for a stroll with a slow pace for 30 minutes" ( $p=0.000)$, "going for a stroll while taking care to not be out of breath" $(p=0.000)$, "doing exercise to the radio or exercise by oneself every day" $(p=0.000)$, "taking a nap every day" ( $p=0.021)$, and "using a mask if someone around oneself gets a cold" ( $p=0.005)$.

The characteristics of groups $\mathrm{A}-\mathrm{C}$ were as follows :

1) Group A : Tolerance to physical activity was high and self-management was near-perfect.

In Group A, 72\% patients fell into NYHA class I and BNP level was $50 \mathrm{pg} / \mathrm{mL}$; that is, cardiac function was maintained, although the median disease duration was the longest (43 months). Compliance with heart failure drugs was significantly high in Group A (29 patients ; $p=0.000)$. Scores of the Health-Related QOL Scale were high for PF $(p=0.027), R P(p=0.034), G H$ $(p=0.007)$, and VT $(p=0.041)$. Regarding scores of the self-care scale, there were significant differences in four items regarding the planning of daily exercise ; the score for "going for a stroll" $(3.66 \pm 1.11)$ was significantly higher in Group A than in groups B ( $p=0.000)$ and C ( $=0.033)$. Score for "going for a stroll while taking care to not be out of breath" $(3.79 \pm 1.05)$ was also significantly higher in Group A ( $p=0.000)$. Scores for "doing exercise to the radio or exercise by oneself every day" $(3.79 \pm 0.94)$ was significantly higher in Group A than in groups B $(p=0.000)$ and $\mathrm{C}(\mathrm{p}=0.001)$. Taken together, patients in Group A were actively planning and engaging in exercise. Regarding adjustments to sleep and rest, score for "taking a nap every day" was significantly lower $(p=0.021)$ in Group A than in Group C, suggesting that Group A patients had the strength to perform continuous activity without rest. Scores for "using a mask if someone around oneself gets a cold" $(4.10 \pm 0.67)$ was significantly higher in Group A $(p=0.005)$, suggesting that patients in this group were actively engaging in infection prevention. Patients in Group A achieved a high capability of performing physical activity and self-care through the activities described above.

2) Group B : Tolerance to physical activity was moderate ; however, patients were not willing to commit to daily exercise and self-care.

Median age of patients in Group B was the highest (76 years).

Table 2. Health-Related QOL Scale for patients with heart failure

\begin{tabular}{lccccc}
\hline & & & & \multicolumn{2}{c}{$\begin{array}{c}\text { Japanese } \\
\text { mean }\end{array}$} \\
\hline Physical Functioning (PF) & 39.94 & $(9.92)$ & 49.84 & $(6.81)$ \\
Role Physical (RP) & 40.49 & $(9.74)$ & 50.07 & $(6.58)$ \\
Bodily Pain(BP) & 50.23 & $(8.41)$ & 50.06 & $(8.55)$ \\
General Health(GH) & 42.16 & $(8.97)$ & 49.96 & $(7.29)$ \\
Vitality (VT) & 44.21 & $(7.79)$ & 50.09 & $(6.83)$ \\
Social Functioning(SF) & 44.52 & $(8.73)$ & 50.00 & $(7.56)$ \\
Role Emotional(RE) & 47.20 & $(6.17)$ & 49.94 & $(6.34)$ \\
Mental Health(MH) & 50.15 & $(6.08)$ & 49.70 & $(7.06)$ \\
\hline
\end{tabular}


Table 3. Scale to Measure Self-Care Behavior of patients with heart falure $\mathrm{N}=70)$

\begin{tabular}{|c|c|c|}
\hline & mean & SD \\
\hline \multicolumn{3}{|l|}{ adjustments to their mental conditions } \\
\hline I try not to be irritated & 4.10 & $(0.59)$ \\
\hline I listen to opinions from others with a calm mind & 4.20 & $(0.44)$ \\
\hline I always act calmly & 4.09 & $(0.65)$ \\
\hline I try not to think too much & 4.04 & $(0.71)$ \\
\hline \multicolumn{3}{|l|}{ planning of daily exercise } \\
\hline I go for strolls & 2.87 & $(1.21)$ \\
\hline I go for a stroll with a slow pace for 30 minutes every day & 2.84 & $(1.26)$ \\
\hline I go for a stroll while taking care not to be out of breath & 3.04 & $(1.28)$ \\
\hline I exercise to the radio or exercise by myself every day & 2.73 & $(1.20)$ \\
\hline \multicolumn{3}{|l|}{ adjustments to sleep and rest } \\
\hline I sleep for at least eight hours & 3.33 & $(1.21)$ \\
\hline I go to sleep before $10 \mathrm{pm}$ & 3.40 & $(1.20)$ \\
\hline I take a nap every day & 2.16 & $(0.73)$ \\
\hline I take a break and get relaxed when doing work and other activities & 4.01 & $(0.79)$ \\
\hline \multicolumn{3}{|l|}{ salt restriction } \\
\hline I add soy sauce or salt when side dishes have little taste $*$ & 3.97 & $(0.70)$ \\
\hline I add soy sauce to pickles $*$ & 3.96 & $(0.69)$ \\
\hline I do not eat salty food & 3.94 & $(0.66)$ \\
\hline I add salt when I eat tempura $*$ & 4.03 & $(0.61)$ \\
\hline \multicolumn{3}{|l|}{ prevention for infection } \\
\hline I wash my hands when I return home or before a meal & 4.11 & $(0.58)$ \\
\hline I use a mask if someone around me gets a cold & 3.74 & $(0.86)$ \\
\hline I brush my teeth and gargle to prevent infection & 3.99 & $(0.71)$ \\
\hline I take a bath to warm my body & 2.39 & $(0.87)$ \\
\hline
\end{tabular}

* : reversal items

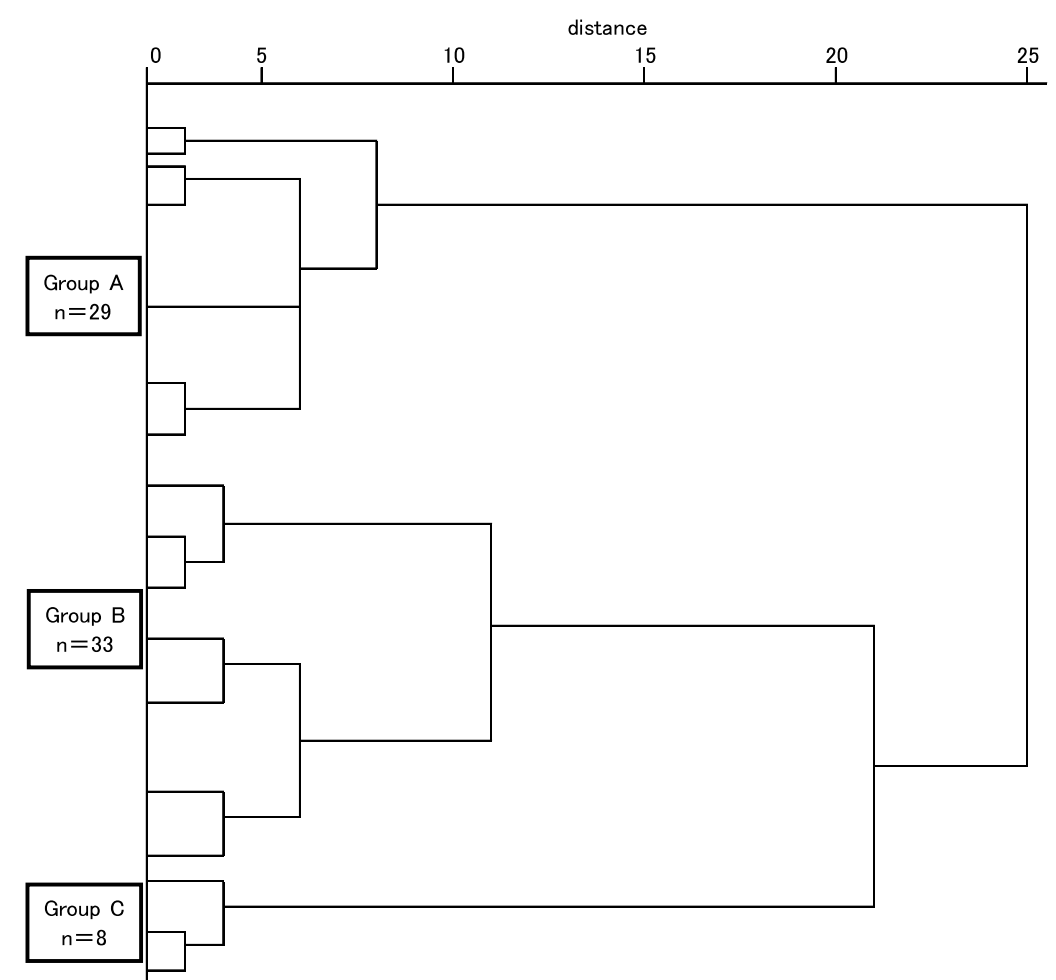

Figure 1. Classification of subjects based on physical activity using hierarchical cluster analysis (Ward's method) 
Table 4. Patient characteristics and clinical symptoms in Group I - III

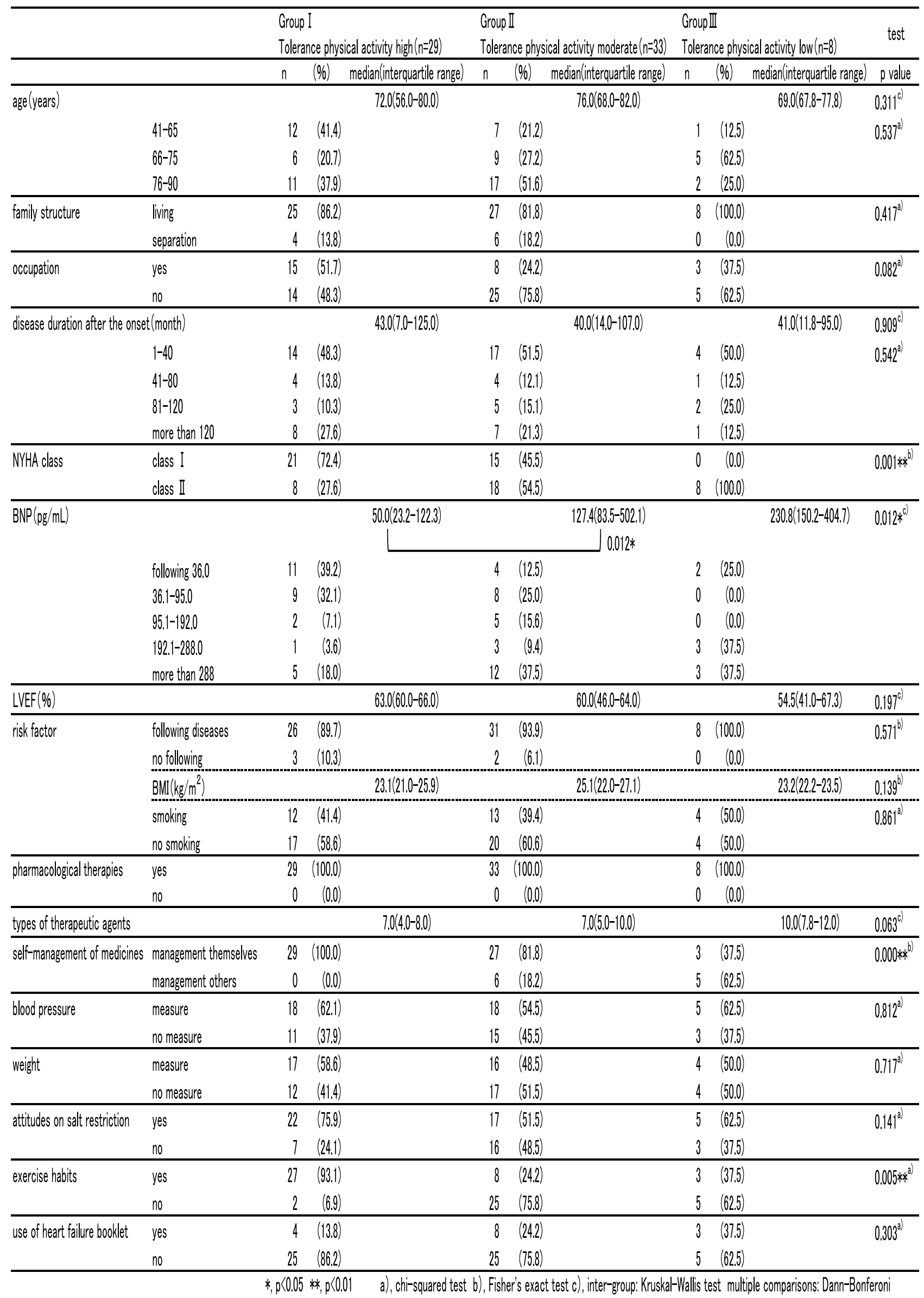


Table 5. Health-Related QOL Scale for patients with heart failure in Group I-III

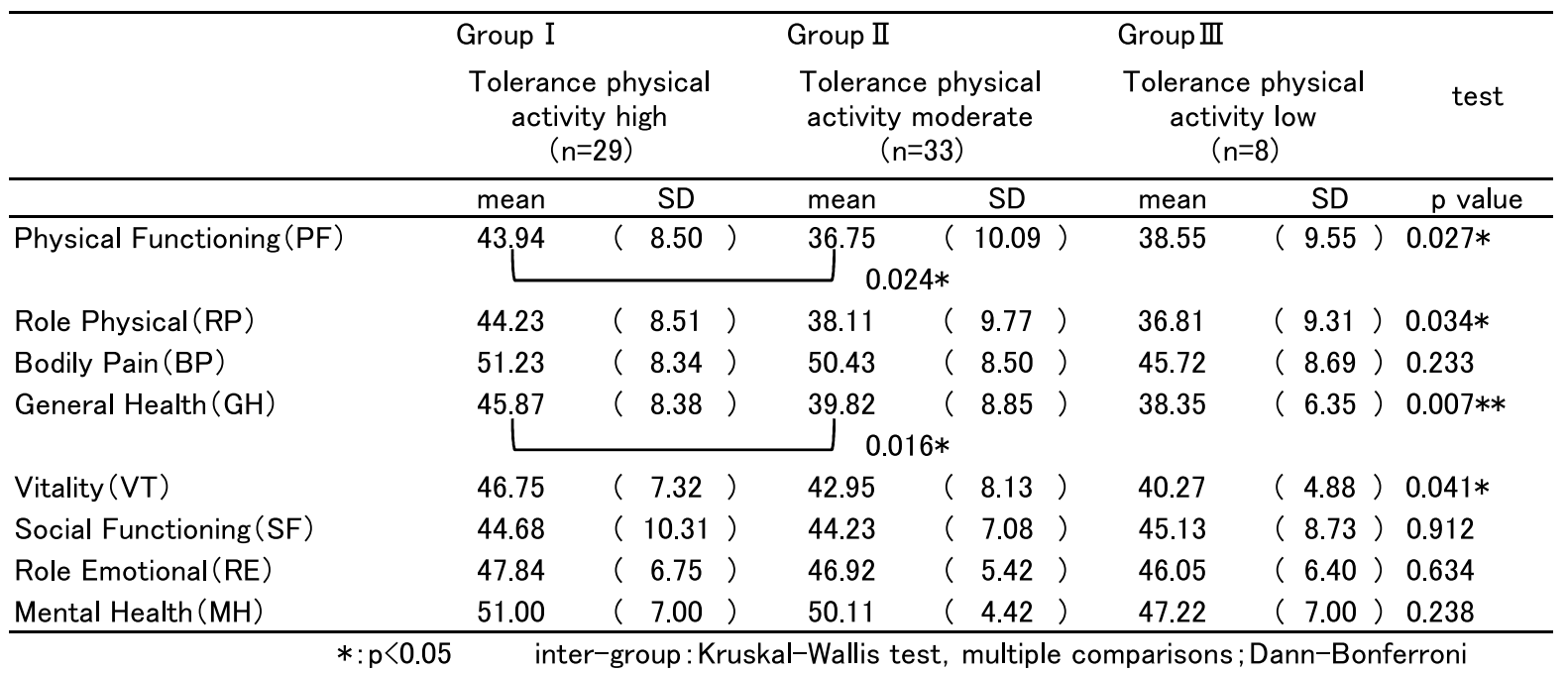

Table 6. Scale to Measure Self-Care Behavior of patients with heart falure in Group I-III

\begin{tabular}{|c|c|c|c|c|c|c|c|}
\hline & \multirow{2}{*}{\multicolumn{2}{|c|}{$\begin{array}{l}\text { Group I } \\
\text { Tolerance physical } \\
\text { activity high } \\
(n=29)\end{array}$}} & \multirow{2}{*}{\multicolumn{2}{|c|}{$\begin{array}{l}\text { Group I } \\
\text { Tolerance physical } \\
\text { activity moderate } \\
\quad(n=33)\end{array}$}} & \multirow{2}{*}{\multicolumn{2}{|c|}{$\begin{array}{l}\text { Group II } \\
\text { Tolerance physical } \\
\text { activity low } \\
(n=8)\end{array}$}} & \multirow{3}{*}{$\begin{array}{l}\text { test } \\
p \text { value }\end{array}$} \\
\hline & & & & & & & \\
\hline & mean & SD & mean & SD & mean & SD & \\
\hline \multicolumn{8}{|l|}{ adjustments to their mental conditions } \\
\hline I try not to be irritated & 4.14 & $(0.58)$ & 4.12 & $(0.60)$ & 3.88 & $(0.64)$ & 0.468 \\
\hline I listen to opinions from others with a calm mind & 4.21 & $(0.49)$ & 4.21 & $(0.42)$ & 4.13 & $(0.35)$ & 0.862 \\
\hline I always act calmly & 4.07 & $(0.75)$ & 4.09 & $(0.63)$ & 4.13 & $(0.35)$ & 0.989 \\
\hline I try not to think too much & 4.00 & $(0.89)$ & 4.06 & $(0.61)$ & 4.13 & $(0.35)$ & 0.991 \\
\hline \multicolumn{8}{|l|}{ planning of daily exercise } \\
\hline I go for strolls & 3.66 & $(1.01)$ & \multicolumn{2}{|c|}{$0.000 * *$} & 2.63 & $(1.19)$ & $0.000 * *$ \\
\hline \multirow[t]{2}{*}{ I go for a stroll with a slow pace for 30 minutes every day } & 3.66 & $(1.11)$ & 2.24 & $(1.03)$ & 2.38 & $(1.06)$ & $0.000 * *$ \\
\hline & & & & & & & \\
\hline I go for a stroll while taking care not to be out of breath & 3.79 & $(1.05)$ & \multicolumn{2}{|c|}{$0.000 * *$} & 2.75 & $(1.16)$ & $0.000 * *$ \\
\hline \multirow[t]{2}{*}{ I exercise to the radio or exercise by myself every day } & $\stackrel{3.79}{L}$ & $(0.94)$ & \multirow{2}{*}{\multicolumn{2}{|c|}{$\int_{0.000 * *}^{1.97}(0.64)$}} & & $(0.93)$ & $0.000 *$ \\
\hline & & & & & & & \\
\hline \multicolumn{8}{|l|}{ adjustments to sleep and rest } \\
\hline I sleep for at least eight hours & 3.10 & $(1.21)$ & 3.64 & $(1.17)$ & 2.88 & $(1.25)$ & 0.117 \\
\hline I go to sleep before $10 \mathrm{pm}$ & 3.10 & $(1.26)$ & 3.61 & $(1.14)$ & 3.63 & $(1.06)$ & 0.238 \\
\hline I take a nap every day & $\stackrel{1}{L}^{1.93}$ & $(0.59)$ & 2.24 & $(0.79)$ & \rfloor$^{2.63} 0.0$ & $0.023 *$ & $0.021 *$ \\
\hline I take a break and get relaxed when doing work and other activities & 4.03 & $(0.82)$ & 4.00 & $(0.87)$ & 4.00 & $(0.00)$ & 0.765 \\
\hline \multicolumn{8}{|l|}{ salt restriction } \\
\hline I add soy sauce or salt when side dishes have little taste $*$ & 3.90 & $(0.82)$ & 3.97 & $(0.64)$ & 4.25 & $(0.46)$ & 0.502 \\
\hline I add soy sauce to pickles $*$ & 3.83 & $(0.89)$ & 4.00 & $(0.50)$ & 4.25 & $(0.46)$ & 0.413 \\
\hline I do not eat salty food & 3.90 & $(0.77)$ & 3.91 & $(0.58)$ & 4.25 & $(0.46)$ & 0.351 \\
\hline I add salt when I eat tempura * & 4.03 & $(0.73)$ & 3.97 & $(0.53)$ & 4.25 & $(0.46)$ & 0.392 \\
\hline \multicolumn{8}{|l|}{ prevention for infection } \\
\hline I wash my hands when I return home or before a meal & 4.24 & $(0.64)$ & 4.09 & $(0.38)$ & 3.75 & $(0.89)$ & 0.097 \\
\hline \multirow[t]{3}{*}{ I use a mask if someone around me gets a cold } & 4.10 & $(0.67)$ & 3.55 & $(0.94)$ & 3.25 & $(0.71)$ & $0.005 * *$ \\
\hline & & & \multicolumn{2}{|c|}{ 」 $0.040 *$} & & & \\
\hline & & & & & & & \\
\hline I brush my teeth and gargle to prevent infection & 4.10 & $(0.72)$ & 3.91 & $(0.72)$ & 3.88 & $(0.64)$ & 0.304 \\
\hline I take a bath to warm my body & 2.52 & $(0.95)$ & 2.30 & $(0.85)$ & 2.25 & $(0.71)$ & 0.513 \\
\hline
\end{tabular}


The proportion of patients who fell into NYHA class I and II was similar. BNP level was $127 \mathrm{pg} / \mathrm{mL}$, which was slightly worse than that in Group A but not as poor as that in Group C. Majority of the patients (76\%) lacked exercise habits. Many patients did not measure blood pressure, although the difference was not significant.

Scores for the Health-Related QOL Scale in PF $(p=0.024)$ and GH $(p=0.016)$ were significantly lower in Group B than in Group A.

Scores for "going for a stroll" $(p=0.000)$, "going for a stroll with a slow pace for 30 minutes every day" $(p=0.000)$, "going for a stroll while taking care to not be out of breath" $(p=0.000)$, and "doing exercise to the radio or exercise by oneself every day" $(p=0.000)$ in the Self-Care Scale were significantly lower in Group B than in Group A. Taken together, patients in Group B showed moderate tolerance to physical activity; however, they were not willing to practice daily exercise and self-care.

3) Group C: Tolerance to physical activity was low, and patients often requested others to handle their self-management, although they were willing to do exercise.

All patients in Group C fell into NYHA class II, and BNP was $230 \mathrm{pg} / \mathrm{mL}$, indicating the worst cardiac function among the three groups. More than half of the patients $(62.5 \%)$ requested others to help with medication management, and $62.5 \%$ patients in this group did not show exercise habits.

Scores for the Health-Related QOL Scale were significantly low for RP ( $p=0.034), \mathrm{GH}(\mathrm{p}=0.007)$, and VT $(\mathrm{p}=0.041)$.

Scores for "going for a stroll with a slow pace for 30 minutes every day" $(p=0.033)$ and "doing exercise to the radio or exercise by oneself every day" $(p=0.001)$ in the self-care scale were significantly lower in Group $\mathrm{C}$ than in Group A but slightly higher than those in Group B, suggesting that Group C patients were exercising. Score for "using a mask if someone around oneself gets a cold" was significantly lower in Group C than in Group A $(p=0.015)$. In contrast, score for "taking a nap every day" was significantly higher in Group C than in Group A $(p=0.023)$ Taken together, Group $\mathrm{C}$ patients showed low tolerance to physical activity, although they were willing to do daily exercise. In addition, they often requested others to manage their self-care.

\section{DISCUSSION}

\section{Summary of patient characteristics}

The Japanese Cardiac Registry of Heart Failure in Cardiology (JCARE-CARD) (16)—an observational study-included patients who were hospitalized due to aggravation of chronic heart failure. The mean age of the patients in that study was 71 years, and $59.7 \%$ patients were males. Moreover, $32 \%$ presented with an ischemic heart disease as the underlying heart disease, and complications such as hypertension $(52.9 \%)$ were prevalent. BMI was $22.3 \mathrm{~kg} / \mathrm{m}^{2}$. These values are similar to those in our cohort ; therefore, our data may be in agreement with the standard and are thus reliable. In addition, we obtained highly reliable data using validated questionnaires [Health-Related QOL Scale (Cronbach's alpha coefficient of 0.65) and Self-Care Scale (Cronbach's alpha coefficient of 0.782)].

\section{Summary of tolerance to physical activity and proposals for tai- lored instruction according to patient conditions}

Normal cardiac function was maintained in Group A patients characterized by high tolerance to physical activity and near-perfect self-care, although the median disease duration was the longest at 43 months. All patients in Group A showed significantly better self-management of medication for heart failure $(p=0.000)$. In addition, scores for the Health-Related QOL
Scale were significantly high for PF $(p=0.027), R P(p=0.034)$, GH $(p=0.007)$, and VT $(p=0.041)$. Patients in Group A were willing to do exercise every day and had the strength to continue activity without rest. Furthermore, they proactively took measures to prevent infection. Although the difference was not significant, they did not restrict salt intake.

Group B patients showed moderate tolerance to physical activity but lacked commitment to daily exercise and self-care. They also showed the highest median age (76 years). Conditions of cardiac function were moderate, and they did not show exercise habits $(p=0.005)$. Scores for the Health-Related QOL Scale for PF $(p=0.024)$ and GH $(p=0.016)$ were significantly low. Scores for all four items related to planning of daily exercise were also significantly low $(p=0.000)$, suggesting that these patients were not actively engaging in physical activity. Patients in this group need to improve activity associated with planning daily exercise and exercise habits.

Group C patients showed low tolerance to physical activity and often requested others to handle their self-management, although they were willing to do exercise; nonetheless, they were the worst among the three groups. Approximately $60 \%$ of the patients requested others to support medication management. They needed to take rest, such as taking a nap, during the day. Scores for the Health-Related QOL Scale were significantly low for RP $(p=0.034)$, GH $(p=0.007)$, and VT $(p=0.041)$. Patients in this group did some exercise ; however, their self-management for protection against heart failure was poor, and they were significantly less likely to use a mask if someone around them gets a cold $(p=0.015)$.

\section{Instructions tailored to the characteristics of heart failure patients in groups $A-C$}

The median disease duration for patients in Group A was relatively long (43 months). They maintained near-normal cardiac function and achieved better self-care, including physical activity, compared with the other groups. First, health professionals should acknowledge patients' appropriate self-care behaviors, such as maintaining physical activity, taking medications and performing blood pressure measurements. However, the scores for salt restriction were the lowest, although the differences were not significant. Poor management of salt restriction and water intake are the most common aspects of symptom control that lead to multiple admissions (4) in patients with heart failure. To avoid such frequent admissions, health professionals should instruct patients regarding the importance of salt restriction and provide dietary counseling on reducing salt intake. In addition, they should instruct patients on the need for continuous self-management, including physical activity, to maintain normal cardiac function and acknowledge it when patients adhere to these instructions to reinforce good self-care behaviors.

The median age in Group B was the highest (76 years), and patients showed moderate tolerance to physical activity but lacked commitment to daily exercise and self-care. They did not plan daily exercise, even though their scores for cardiac function were within an acceptable range. A previous study showed that muscular weakness in the lower limbs restricts physical activities (17). Another study demonstrated that exercise training is required to improve tolerance to physical activity in patients with heart failure (18). Physical therapists and other health professionals should proactively recommend exercise training and easy calisthenics, such as exercise to the radio at home, to patients without exercise habits. Furthermore, these professionals should actively provide information related to exercise to make exercise a daily habit.

Scores for cardiac function were the worst in Group C, and patients showed low tolerance to physical activity and often 
requested others to handle their self-management, although they were willing to do exercise. Patients in Group C used ten types of heart failure drugs, a number higher than that in the other groups. Several studies have indicated a number of aspects of poor medication adherence, which include one or more of the following : missing doses, confusion caused by taking several drugs, and incapacity to maintain medication adherence $(19,20,21)$. More than half of the Group C patients (60\%) asked others to handle their medication management. Health professionals should provide a method that enables such patients to achieve better medication adherence. In addition, personalized instructions should be provided. We believe that re-education on the importance of using a mask is effective in this group in order to prevent infections (3), which is a factor causing aggravation of heart failure. To prevent worsening symptoms, recording daily life self-management status into Shinfuzen Techou [Heart Failure Booklet] (22) and supporting patients through telephone interviews (23) are effective. We intend to promote opportunities to study personalized medication adherence and behaviors to prevent the occurrence of infection.

\section{Common proposal for three groups of heart failure patients}

The smoking rate was high in common among the three groups. Smoking is a risk factor for heart failure patients and is involved in physical activity. Therefore, it will be explained by emphasizing that it is a risk factor. In addition to physical activity in normal life, we would like to propose an education that encourages re-education, such as affecting circulation function and reducing the recurrence rate and mortality rate by quitting smoking.

Not only during hospitalization but also at outpatient consultations, we would like to make sure that we can continue to establish knowledge and maintain self-management, and to repeat education, according to individual circumstances. We would like to propose individual guidance not only to individual patients but also to patient families and to give group classrooms and lectures in workplaces and communities.

This study included patients with heart failure classified into NYHA class I and II ; therefore, the results of our study cannot be generalized to all heart failure patients. We used a common tool to assess cardiac function ; thus, cardiac function itself was not evaluated. Furthermore, no analysis was performed on the doses of medications for heart failure. Taken together, longitudinal studies are warranted to address these issues in the future.

\section{$\mathrm{COI}$}

The authors have no conflicts of interest directly relevant to the content of this article.

\section{ACKNOWLEDGEMENTS}

The authors would like to thank all the staff members and patients of the cooperating hospital for participation.

\section{REFERENCES}

1. Makaya M, Tsutsui $\mathrm{H}$ : Disease management in patients with chronic heart failure from the study of team medicine. Japanese journal of cardiovascular disease prevention 44(3) : 194-199, 2009

2. Ministry of Health, Labour and Welfare: Overview of the 2017 patient survey, https://www.mhlw.go.jp/toukei/saikin/ hw/kanja/17/index.html (2019.9.15)

3. The Japanese Circulation Society : Guidelines for diagnosis and treatment of acute and chronic heart failure (revised in 2017), http://www.j-circ.or.jp/guideline/pdf/JCS2017_tsutsui_h.pdf (2018.6.4)

4. Makaya $\mathrm{M}$ : Disease management program for improvement of prognosis and quality of life in patients with heart failure. The journal of Japanese Association of Cardiovasucular Nursing 4(1) : 13-17, 2008

5. Ohkawa $\mathrm{T}:$ Response to chronic heart failure exacerbation cases repeatedly entered and discharged effects of response by heart failure home support team. Journal of Japan Cardiac Rehabilitation 10(2) : 272-276, 2005

6. Kitamura M, Saitoh K, Mizutani M, Yokoi T, Yaekura M, Mimura Y, Nagashima H : Recovery Process of Mobility and Prognosis of Walking Independence in Elderly Patients with Heart Failure. The Journal of Japanese Physical Therapy Association 43(1) : 47-55, 2015

7. Nagaoka S, Uchida Y : Physical, psychological and living conditions of older patients with heart failure and necessary care at discharge and 2 months after discharge- Examination of support for safe and comfortable life at home-. Annals of Gunma University Graduate School of Health Sciences 32 : 61-69, 2011

8. Agawa K, Hara S, Ono M : Awareness of Physical Change in Their Daily Lives among Elderly Patients with Chronic Heart Failure. Journal of Japan Academy of Gerontological Nursing 17(1) : 46-54, 2012

9. Robert R, Louise M, Andrew P, William D, Lyall H, Andreas W, Stephen L, Paul M, Ronald P, Kerry C, Neil O, Louise B, Sophia P, Monika D, Heather T, Chris B : Determinants of physical activity after hospitalization for coronary artery disease : the Tracking Exercise After Cardiac Hospitalization (TEACH) Study. European journal of cardiovascular prevention and rehabilitation, official journal of the European Society of Cardiology Aug 13(4) : 529-537, 2006

10. The Japanese Circulation Society: Guidelines for the treatment of chronic heart failure (revised in 2010), http:// www.j-circ.or.jp/guideline/pdf/JCS2010_matsuzaki_h.pdf (2017.7.8)

11. Moriyama M, Tsuge N, Furui Y, Nakano M, Takyu H, Iwamoto S : A survey of current status of patient education and willingness to use disease management service programs in hospitals and clinics in Japan. Journal of the Japanese Society on Hospital Administration 43(1) : 47-58, 2006

12. Fukuhara $\mathrm{S}$, Suzukamo $\mathrm{Y}$ : Instruments for measuring Health-related Quality of Life -SF-8 and SF-36. Journal of clinical and experimental medicine 213(2) : 133-136, 2005

13. Fukuhara S, Suzukamo Y : Manual of the SF-8 Japanese version Institute for Health Outcomes\& Process Evaluation Research, Kyoto, 2004

14. Sasayama S, Asanoi H, Ishizaka S, Miyagi K : Evaluation of functional capacity of patients with congestive heart failure. New Aspects in the treatment of failing heart, Springer Verlag, Tokyo, 113-117, 1992

15. Yoshie Y, Takama S : The development and Trial of scale to measure self-care behavior of patients with heart disease. The journal of the Nursing Society of University of Toyama 14(1) : 91-99, 2014

16. Tsutsui H, Tsuchihashi M, Kinugawa S, Goto D, Takeshita A : Clinical characteristics and outcome of hospitalized patients with heart failure in Japan - rationale and design of Japanese Cardiac Registry of Heart Failure in Cardiology (JCARE- CARD)—, Circulation Journal $70:$ 1617-1623, 
2006

17. Takeichi N, Izawa K, Watanabe S, Hiraki K, Morio Y, Osada N, Omiya K : Relationships between physical activity and Lower extremity muscle strength at entry into recovery phase by patients with coronary artery disease. The Journal of Japanese Physical Therapy Association 36(3) : 109 113,2009

18. Kinugawa S : Special Issue Cardiac Rehabilitation From perioperative management to long-term prognosis improvement 6. Pathophysiology and Exercise Therapy for Heart Failure Why Is It Necessary? Why is it valid? How do I do that? Official journal of Japan Society of Circulation Control in Medicine 38(2) : 113-118, 2017

19. Tase Y, Hashimoto Y, Umezu T, Ito K,Muto M : Factors of lack of self-care in patients with heart failure who repeatedly enter and leave the hospital. Journal of the Japanese Society of Nursing 2 Adult Nursing $34: 141-143,2003$

20. Naito M, Hatamoti $\mathrm{T}$ : The actual situation of self-care in elderly heart failure patients : A case study of patients living alone and family living together. Hāto nāshingu 20(12) : 1246-1251, 2007

21. Naya $\mathrm{T}$ : Current situation of self-care and nursing care for outpatient 75 or older with chronic heart failure. Journal of Japan Academy of Gerontological Nursing 13(1) : 40-48, 2008

22. Nagano Y, Suehiro M, Okamura M, Hataoka H, Ichiba M, Watanabe M, Ikezoe S : Nursing care supporting life adjustment for elderly patients with chronic heart failure. Journal of Kochi Women's University Academy of Nursing 42(2) : 8796, 2017

23. Shiokumi N, Sawatari H, Miyazono M, Hashiguchi N, Higuchi M, Nakamura T, Yoshitake S, Tashiro H, Yamasaki K, Izukura R, Magota C, Kuroda H, Fujita K, Chishaki A : Effects of telephone supports on promoting depression and QOL of outpatients with chronic heart failure -Analysis of different support periods-.Japanese journal of cardiovascular disease prevention 51(3) : 157-165, 2016 